\title{
MORRIS WATER MAZE - A BENCHMARK TEST FOR LEARNING AND MEMORY DISORDERS IN ANIMAL MODELS: A REVIEW
}

\author{
CH. VENKATARAMAIAH, G. SWATHI, W. RAJENDRA*
}

Division of Molecular Biology, Department of Zoology, Sri Venkateswara University, Tirupati, Andhra Pradesh, India. Email: rwudayagiri16@gmail.com

Received: 12 December 2017, Revised and Accepted: 24 January 2017

\section{ABSTRACT}

The morris water maze (MWM) was developed by morris as a device to investigate spatial learning and memory in laboratory rats. MWM has become one of the most frequently used laboratory tools in behavioral neuroscience. The MWM task has been often used in the validation of rodent models for neurocognitive disorders (e.g., Parkinson, Alzheimer, Epilepsy, and Schizophrenia), and the evaluation of possible neurocognitive treatments. It is also being used to assess the properties of established potential antipsychotics in animal models of Schizophrenia. The MWM task requires rats to find a hidden platform in a large, circular pool of water that is colored opaque with powdered non-fat milk (or) non-toxic tempera paint where they must swim to the hidden platform. Because they are in the opaque water, the animals cannot see the platform and cannot rely on scent to find the escape route. Instead, they must rely on extra-maze cues. The behavior of rat can be evaluated by analyzing the different parameters such as escape latency, swim speed, and path length, and probe trail. The purpose of this review is to briefly describe procedural aspects, interpretational difficulties of data and advantages of MWM. This paradigm has become a benchmark test for learning and memory difficulties in animal models and preclinical research in general.

Key words: Learning and memory deficits, Animal models, Morris water maze, Data analysis.

(C) 2018 The Authors. Published by Innovare Academic Sciences Pvt Ltd. This is an open access article under the CC BY license (http://creativecommons. org/licenses/by/4. 0/) DOI: http://dx.doi.org/10.22159/ajpcr.2018.v11i5.24292

\section{INTRODUCTION}

Morris water maze (MWM) task is classic and probably most widely used test of spatial learning and memory. The apparatus was designed by Morris in 1981 [1]. It represents a circular maze with larger diameter $(180 \mathrm{~cm})$, filled with opaque water, in which a rodent (typically a rat) collected from various locations and released on the pool periphery, reaches for an escape platform hidden under the water surface. In addition, the test uses the natural ability of rats to swim while not causing major distress to the animals despite a significant stress response induced by the task [2,3]. Another advantage of this test is its simplicity, which has contributed significantly to its success and massive use. The MWM is typically used in two basic experimental protocols such as the reference memory version and delayed matching to place [4-6]

\section{The reference memory version}

It involves the training of rats released from the periphery of the pool to find a hidden platform, the position of which is stable across daily sessions. There are visually more swims in daily sessions. An early study by Morris showed that place navigation in this version is impaired by a hippocampal lesion. However, this version can also be made hippocampus-dependent as well as resistant to several pharmacological manipulations after extensive pre-training (or) specific training protocols. An interesting procedure is the so-called non-spatial pre-training in which animals are first made familiar with all procedural aspects of the task, such as the platform is in the maze [7]. It is pursued by closing a dark curtain around the pool (without any cues) and putting the submerged platform is random places in the pool. Animals thus get fully acquainted with searching for the platform but do not know where to reach. Such pre-training was found to eliminate a number of cognitive deficits such as NMDA receptor and cholinergic blockade [7,8] serotonin depletion [9], and diazepam treatment [10].

\section{Delayed matching to place version}

In which animals are released in four trials a day, and the platform position is changed in between the days (but always stays the same within four on a particular day). Therefore, the rat cannot locate the platform correctly (or) find it just by chance in the first swim every day. The second swim already contains a memory trace from the first one, and typically this swim is evaluated. Moreover, multiple intertrial intervals between first and second swims can be used. (e.g., $15 \mathrm{~s}$, $20 \mathrm{~min}$ ). There should be no interface between positions in a particular day. (Each position should be used only once). The task represents the paradigm for assessment of one trial spatial learning. The short delays may be used for evaluating the persistence of the memory trace [6]. The delayed-matching-to-place test in the MWM is strictly hippocampusdependent, which means that the animal with a hippocampal lesion was not capable of improving from first to the second swim and thus failed to learn even after extensive experience with the maze [5].

The MWM task has become a benchmark test for learning and memory deficits in animal models and preclinical research in general. In the contest of the use of this test, it might be emphasized that there are also other modifications and protocols including a working memory version, cognitive flexibility, neuroprotection, cholinergic dysfunction, and path integration in the MWM [11-16]. Testing the working memory in MWM is required due to information processing and working memory deficits in many central nervous system disorders, such as Schizophrenia, Parkinson, and Alzheimer's [17]. MWM is widely used to assess the pharmacological properties of established potential antipsychotics in animal models of schizophrenia which impair memory in the MWM test in naïve rats [18-21]. The MWM test can also be used as a tool to find out the efficacy of biomolecules and Psychotropic drugs on chemically induced effects of learning and memory in rodent models [22,23]. Keeping in view of the relative importance of MWM Task, in this review, we have discussed the apparatus setting, procedure, data recording methods, swim patterns and strategies, factor and data analysis, limitations and interpretational difficulties and criticisms of MWM, used for testing learning and memory disorders in rodent models.

\section{METHOD}

Apparatus setting[24].

- The maze was constructed out of a circular polypropylene pool that measured $110 \mathrm{~cm}$ in diameter and $20 \mathrm{~cm}$ in depth. 
- A $1.5 \mathrm{~m}$ diameter, $45 \mathrm{~cm}$ deep MWM was filled with water to a depth of $26.5 \mathrm{~cm}$ at room temperature.

- The water was made opaque with the addition of $500 \mathrm{mlnon}$-toxic white liquid tempera paint to ensure camouflage of the white escape platform.

- The pool was filled with water until it had reached a height of about $5 \mathrm{~cm}, 100 \mathrm{ml}$ of white liquid tempera paint was mixed in about $25 \mathrm{ml}$ of water.

- The diluted paint concentrate was then poured into the pool. Subsequently, water was again added to the pool until the platform is submerged by $1 \mathrm{~cm}$ of whitewater.

- The water was left to sit overnight to achieve room temperature. $\left(22 \pm 1^{\circ} \mathrm{C}\right)$.

- The pool was divided into four quadrants. Such as ;"north-west," "northeast," "southwest," "southeast." Boundaries of the quadrants were measured on the edges of the pool with marking tape and labeled as north, south, east, and west.

- A plexiglas cylinder (13.75*9 cm diameter) was used as the escape platform in the maze. The cylinder was filled with water to weight it down in the pool.

- The platform had a removable red and yellow stripped top $(3 \mathrm{~cm} *$ $9 \mathrm{~cm}$ in diameter) with a colorful flag erected in the center.

- The level of the water in the pool was adjusted to within $0.5 \mathrm{~cm}$ below the surface of the stripped top thus creating the visible escape platform (or) to $0.5 \mathrm{~cm}$ above the white cylinder (without stripped top), thus creating an invisible escape platform (Fig. 1).

The room in which the testing apparatus is located contains environmental cues, which aid the rodent in spatial learning. These cues may be high contrast geometric patterns placed on the walls of the room, posters or anything visible to the animal from the water and platform, such as room dividers, equipment, furniture, and door frames. The performance of the animal in the MWM task was tracked using a video-camera based computer tracking system (water maze beta, Actimetrics software), on IBM PC computer, with a camera fixed to ceil $2.1 \mathrm{~cm}$ above the pool.

\section{Test conditions [25].}

- The water temperature was adjusted according to the age of the rats: $29^{\circ} \mathrm{C}( \pm 0.5)$ for postnatal day (PND) $16-20 ; 27^{\circ} \mathrm{C}( \pm 0.5)$ for PND $20-27$ and $25^{\circ} \mathrm{C}( \pm 0.5)$ for adult rats.

- Immediately before behavior testing, the rats were allowed an adaptation period of 10-15 min (to adapt to white light).

- The rats were placed on the platform for $1 \mathrm{~min}$ before undergoing three swim training trials.

- During each training trail, the rat was removed from the stand and released facing the platform at a distance of 12-18 inches.

- The rat was then guided by the experimenter to swim to the platform.

- A rest period of the $30 \mathrm{~s}$ was followed by 12 test trails: Two sets of 6 trails - 4 trails from each of the 4 start positions, with a rest period of 30-45 min in a small heated cage in a different room (separating the test periods).

- During the test trial, the rats were released into the water at one of the four starting positions, facing the wall and were required to use distal cues to navigate to the platform.

- The start positions were all equidistant from the platform, located immediately adjacent to the wall in the center of the four quadrants that did not contain the platform.

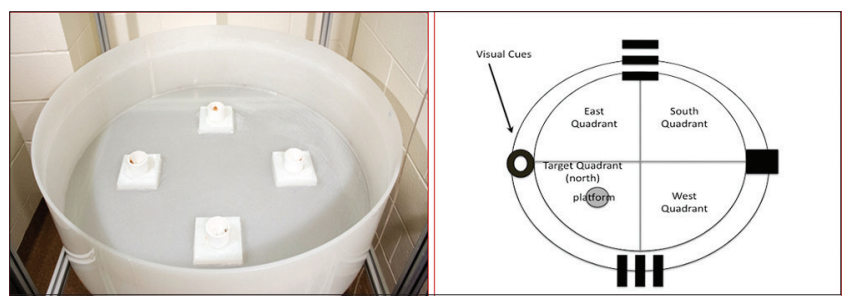

Fig. 1: Apparatus and quadrant poles of morris water maze. High contrast visual cues should be used. Rodents cannot see color and do not have good visual acuity
- The rat was allowed to swim for up to $1 \mathrm{~min}$ to locate the platform. If it failed to locate the platform within that time, escape was assisted.

- Pups (PND 16-20) were given a 60 s intertrial interval on the platform. While older rats ( $\geq 20$ days) were given a $35 \mathrm{~s}$ intertrail interval.

- Distance traveled and escaped latency were measured on each trail. Swim speeds, in $\mathrm{cm} / \mathrm{s}$, were calculated by dividing the distance traveled by the escape latency.

\section{PROCEDURE}

Testing in the MWM lasted 8 days. The $1^{\text {st }} 3$ days were acquisition training with an invisible platform. Days 4-6 were reversal training, again with an invisible platform. On the $7^{\text {th }}$ day, a probe trial was conducted with no escape platform. On day 8, 4 trails were conducted using the visible platform.

\section{Running rat}

During the test period, the rat was placed in a clean empty cage (no bedding). Paper towel was placed on the bottom of the cage to allow the mice to dry more quickly. This paper towel was replaced when it becomes completely wet. Rats were run in squads of 5-6 with $10 \mathrm{~min}$ between each trail for each animal.

\section{Acquisition}

During acquisition training, the water was adjusted approximately such that the platform was covered by $1 \mathrm{~cm}$ of water (visible platform). The platform was placed in the center of North West quadrant. Each animal received 4 trials of $60 \mathrm{~s}(\max )$ per day. The starting positions of the animals were predetermined which prevent any sequence of 2 trials to be repeated by the same animal during any other day. Possible start positions were at the boundaries of the quadrants. (e.g., west, north, east, or south.). Each rat was removed from its holding cage using a small, clean $500 \mathrm{ml}$ plastic yogurt container to minimize handling stress. The animal was then placed into the water at the appropriate start position, facing the center of the pool. The mouse was then permitted to explore the pool and to search for the hidden escape platform for $60 \mathrm{~s}$. When the animal located the platform, the timer was stopped, and the animal was removed using the plastic container and placed in the holding cage. If the animal did not find the platform during the allotted time, the animal was guided onto the platform using the plastic container. Once on the platform, the animals were permitted to visually explore their surroundings for $20 \mathrm{~s}$ at which point, the researcher picked up the rat in the plastic container and returned the animal to the appropriate holding cage. The next animal was then placed in the pool, and the same procedure was followed. Each animal was permitted 4 trails per day over 3 days, for 12 trails of acquisition training.

\section{Reversal}

Reversal training began on day 4. The visible platform was moved to the opposite quadrant (south-east quadrant), and rats were again assigned to appropriate start positions. The procedures as in acquisition training were again carried out during reversal training. Each animal was permitted 4 trails for day for 3 days for a total of 12 trails of reversal training. During acquisition and reversal training the following behaviors were measured. Such as

- Swim latency (time to find out and mount the escape platform),

- Swim distance,

- Average velocity

- Duration and frequency of Thigmotaxis behavior

- Swim path error and proximity to platform.

\section{Probe trial}

A probe trial was conducted on the day 7. At this time, there was no escape platform in the maze at all. Each animal completed one trail of $60 \mathrm{~s}$. Each rat was placed in the maze from one of the four possible start positions and allowed to explore the pool. During the probe trial, the following measures were recorded.

- Duration of time spent in each quadrant (north-east, south-east, north-west, and south-west). 
- The number of times the mouse crossed the location of the platform during acquisition training (annulus reversal crossing).

- The number of times the mouse crossed the location of the platform during acquisition training (annulus acquisition training)

\section{Visible platform}

The visible platform task was conducted on day 8. The visible platform was placed in the south-west quadrant of the pool. The same procedures as in acquisition and reversal training were carried out, and the animals were allowed to complete 4 trails.

Swimming error is measured as the inability of mouse to swim in a relatively direct path from the start position to the location of the hidden (absent) platform [26]. A correct score (assigned a value of 0) was obtained when the subject swam directly to the platform while remaining within a 9-cm corridor, extending from the start location to the platform. Any deviation from a direct swim path in a relatively straight line within the corridor resulted in an incorrect score (given a value of 1). The proximity measure with respect to the escape platform was calculated by sampling the position of the subject in the pool ( 5 times per second) to provide a good record of its distance from the escape platform in 1-s averages [27]. By this method, the proximity scores were obtained, which reflect search error (deviations from an optimal search)

\section{DATA RECORDING}

\section{Manually}

Search paths can be obtained by placing transparent plastic sheets over a television screen and tracking the movement of each rat with a marker. The distance traveled, and the diameter of the maze on the plastic transparencies (scales and plan wheel XL) can then be used to determine the distance traveled in the actual maze using the formula [(real maze diameter/transparent sheet diameter) $\times$ tracking distance]. With probe trials, transparent plastic sheets were placed on a television screen and lines were drawn on the maze (as seen on the T.V.), so as to divide it into four equal quadrants. The duration in each zone was then scored by playback of video recorded trails using hindsight computer software (MS-Dos 1.5).

\section{Automated}

Search paths, distance traveled, average velocity, proximity measures, and search error can be obtained using a video camera based tracking system and the water maze beta (Acti metrics, http://www.actimetrics. com)software). The system tracks the movement of each animal throughout the trail and auto-calculates the measures of interest.

\section{FACTOR ANALYSIS}

Wolfer et al. (1998) found three factors which accounted for 81\% of the observed variability in the scores of mice in the MWM task: Thigmotaxis (49\%), passivity (19\%), and memory (13\%). Thigmotaxis is associated with swimming along the walls of the pool; while passivity means floating in the pool, with a slow swimming speed; and memory represents the time spent in the target quadrant during the probe trail [28] (Fig. 2).

\section{DATA ANALYSIS}

During the data analysis, the following parameters were measured. Such as

- Distance moved

- Mean velocity

- Time in each quadrant

- Percent time in each quadrant

- Escape velocity

- Thigmotaxis

- For each day and each mouse, average the 5 trials to give a single path length and escape latency for each test subject. Calculate the combined error appropriately. For day 6, simply collect the path length, escape latency, and time spent in the platform quadrant for each mouse

- If any differences exist between groups in day 1 , it is likely a problem with vision rather than learning and memory. Only proceed with analysis if no differences are seen in day 1 .

- Compare the learning curves for days 2-5 using statistics appropriate for your data set. A steeper curve represents faster task acquisition; a shallower curve represents a deficit in task acquisition. The data from day 2 to day 5 are analyzed using ANOVA

- For day 6, compare the percent of time spent in the previously learned platform quadrant, using statistics appropriate for your data set. A higher percentage of time spent in the platform quadrant is interpreted as a higher level of memory retention.

\section{SOME COMMON CONFOUNDS IN THE WATER MAZE}

- The treatment condition (drug, age, etc.) impairs the animals' vision, search strategy, stamina, etc.

- The treatments alter the motivation to escape from the water.

- Behavioral despair or anxiety is induced

- Some animals have more effective or efficient search strategies

- Hypothermia may alter behavior differentially in different treatment groups.

- Motor coordination or stamina may be differentially affected in different treatment groups.

- Different groups of animals do not have the same baseline behavior (i.e., do not have the same final learning times, the same rate of learning, or the same performance during invisible platform trials)

- The variability within subjects is high often enough to lose all statistical power. Cohort to cohort variability is also high

- Water temperature can affect performance.

\section{LIMITATIONS AND INTERPRETATIONAL DIFFICULTIES}

Interpretation of results in MWM suffers drawbacks as the performance of subjects in the MWM can be significantly influenced and interfered by a variety of technical as well as procedural variables such as dimensions of the pool, the water temperature [28], strain and sex of rats [29], different schedule of training [30], task parameters [31], aging stress [32], prenatal nutritional status [33], postnatal nutritional status and hormonal status [34], day of oestrus cycle [35], body temperature [36], and home care environment [37]. The immersion of the animals into the water and the initial sensation of being trapped in it may cause considerable stress during the first stages of the test [38].

\section{CRITICISM}

The swim path analysis can be used to determine whether rats are developing a spatial strategy for learning the maze or are using nonspatial strategies, such as circular swimming or Thigmotaxis. Analysis of swim speed may be necessary to control for locomotor differences between strains [39].

\section{CONCLUSION}

The MWM test has become one of the most frequently used research tool in various subfields of behavioral neuroscience. The test has the number of advantages and has been used in such an impressive variety of applications that it might also rank as one of the best assays for spatial learning and memory in laboratory rodents. The relative simplicity of the MWM task undoubtedly one of the reason for its continuing success, also the possibility to differentiate between the spatial (hidden-platform) and non-spatial (visible-platform) conditions of the task and the task can be altered in numerous ways to investigate working memory, reference memory and task strategy [40]. The robustiousness/simplicity and reliability make this paradigm as one of the "gold standards" of behavioral neuroscience. Although it may continue to be a useful research tool for many years to come, its disadvantages and limitations should be recognized as well, and researchers should continue to look for new and better alternatives. 


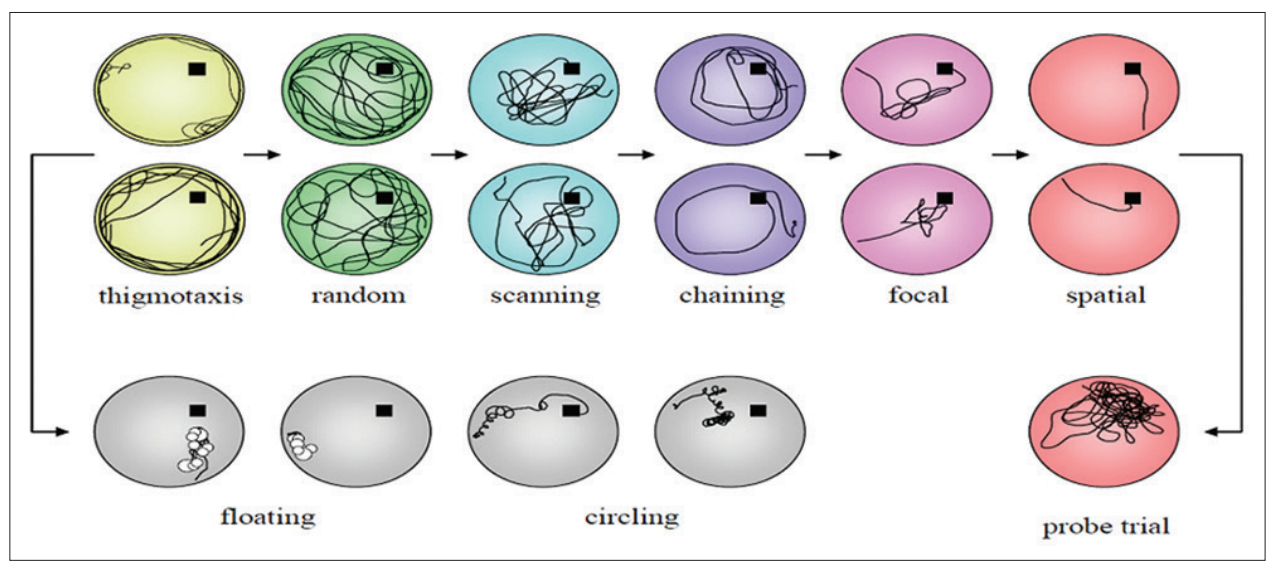

Fig. 2: Reference strategies and swim patterns in the morris water maze

\section{ACKNOWLEDGMENTS}

Mr. Ch. Venkataramaiah is grateful to the Department of Science and Technology, New Delhi for Providing Financial assistance through the award of an Inspire Fellowship.

\section{AUTHORS CONTRIBUTIONS}

The authors contributed equally to this work.

\section{CONFLICTS OF INTEREST}

The authors declare that they have no conflict of interest.

\section{REFERENCES}

1. Morris RG. Spatial localization does not depend on the presence of local cues. Learn Motiv 1981;12:239-60

2. Hölscher C. Stress impairs performance in spatial water maze learning tasks. Behav Brain Res 1999;100:225-35.

3. D'Hooge R, De Deyn PP. Applications of the morris water maze in the study of learning and memory. Brain Res Brain Res Rev 2001;36:60-90.

4. Clark RE, Martin SJ. Interrogating rodents regarding their objects and spatial memory. Curr Opin Neurobiol 2005;15:593-8

5. Steele RI, Morris RG. Delay-dependent impairment of a matching-toplace task with chronic and intra hippocampal infusion of the NMDAantagonist D-AP5. Hippocamp 1999;9:118-36.

6. Carroll CM, Martin SJ, Sandin J, Frengueli B, Morris GM. Dopaminergic modulation of the persistence of one-trail hippocampusdependent memory. Learn Mem 2006;13:760-9.

7. Saucier D, Hargreaves EL, Boon F, Vanderwolf CH, Cain DP. Detailed behavioral analysis of water maze acquisition under systemic NMDA or muscarinic antagonism: Nonspatial pretraining eliminates spatial learning deficits. Behav Neurosci 1996;110:103-16.

8. Cain DP, Saucier D, Boon F. Testing hypotheses of spatial learning: The role of NMDA receptors and NMDA-mediated long-term potentiation. Behav Brain Res 1997;84:179-93.

9. Beiko J, Candusso I, Cain DP. The effect of non-spatial water maze pretraining in rats subjected to serotonin depletion and muscarinic receptor antagonism: A detailed behavioral assessment of spatial performance. Behav Brain Res 1997;88:201-11.

10. Cain DP. Prior non-spatial pre-training eliminates sensorimotor disturbances and impairments in water maze learning caused by diazepam. Psychopharmacol 1997;84:1769-93.

11. Rezacova L, Svoboda J, Stuchlik A, Vales K. Differential effects of stable elevated levels of corticotropin-releasing hormone and systemic corticosterone on various types of rat learning. Neuro Endocrinol Lett 2011;32:64-76

12. Bheemesh V,PoornimaAM,HegdeA,KumarA.Pterocarpus Marsupium heartwood extract restores learning, memory and cognitive flexibility in an STZ-Na Induced Diabetes Animal model. Int J Pharm Pharm Sci 2016;8:339-43.

13. Shelat DY, Acharya AR. Neuroprotective activity of novel cur-ca-thione and its oxidative stress study. Int J Pharm Pharm Sci 2016;8:167-73.

14. Awasthi H, Siddiqui HH. Candesartan reverses memory deficit caused by colchicine-induced cholinergic dysfunction and oxidative stress. Int
J Pharm Pharm Sci 2017;9:184-9

15. Spooner RI, Thomson A, Hall J, Morris RG, Salter SH. The atlantis platform: A new design and further developments of Buresova's ondemand platform for the water maze. Learn Mem 1994;1:203-11.

16. Benhamou S. Path integration by swimming rats Anim Behav 1997; $54: 321-7$

17. Van Snellenberg JX. Working memory and long-term memory deficits in schizophrenia: Is there a common substrate? Psychiatry Res 2009; $174: 89-96$

18. Bubeníková-Valesová V, Horácek J, Vrajová M, Höschl C. Models of schizophrenia in humans and animals based on inhibition of NMDA receptors. Neurosci Biobehav Rev 2008;32:1014-23.

19. van der Staay FJ, Rutten K, Erb C, Blokland A. Effects of the cognition impairer MK-801 on learning and memory in mice and rats. Behav Brain Res 2011;220:215-29.

20. Agnes Fonseca RF, Vania Eloisa DA, Yone DeAN, Djenane Ramalho DO. Use of psychotropics in everyday life from the perspective of health professionals and patients: A systematic review. J Crit Rev 2017;4:1-8.

21. Skarsfeldt T. Differential effect of antipsychotics on place navigation of rats in the MWM. A comparative study between novel and reference antipsychotics. Psychopharmacol 1996;124:126-33.

22. Niraj Kumar JH, Kumar P. Molecular docking studies for the comparative analysis of different biomolecules to target hypoxiainducible factor-1 $\alpha$. Int J Appl Pharm 2017;9:83-9.

23. Madhavi E, Gopala Rao KV, Ruckmani A, Umar D. Evaluation of antiepileptic activity of ethanolic extract of Azima tetracantha root in mice. Int J Curr Pharm Res 2016;8:76-9.

24. Paylor R, Crawley JN. Inbred mouse strain differences in the prepulse inhibition model of schizophrenia. Psychopharmacol 1997;3:23-8.

25. Shultz T, Tonkiss J, Galler JR. An analysis of spatial navigation in prenatally protein malnourished rats. Physiol Behav 1994;55:147-78.

26. Whishaw IQ. Formation of a place learning-set by the rat: A new paradigm for neurobehavioral studies. Physiol Behav 1985;35:139-43.

27. Gallagher M, Burwell R, Burchinal M. Severity of spatial learning impairment in aging: Development of a learning index for performance in the morris water maze. Behav Neurosci 1993;107:618-26.

28. Kikusai T, Tonohiro T, Kaneko T. Simultaneous evaluation of spatial working memory and motivation by the allocentric place discrimination task in the water maze in rats. J Vet Med Sci 1999;61:673-81.

29. Cimadevelli JM, Gonzalez-Pardo H, Lopez L, Diaz F, Cueto EG, Moreno LM, et al. Sex-related differences in spatial learning during the early postnatal development of the rat. Behav Process 1999;46:159-71.

30. Kanit L, Yilmax O, Tskiran D, Balkan B, Furedy J, Pogus J. Intersession interval affects performance in the Morris Water Maze. Int J Neurosci 1998;96:197-204.

31. Roof RL, Stein DG. Gender differences in morris water maze performance depend on task parameters. Physiol Behav 1999;68:81-6.

32. Aleksandov AA, Polyakova ON, Bateuv AS. The effects of prenatal stress on learning in rats in a Morris maze. Neurosci Behav Physiol 2001;31:71-4

33. Tonkiss J, Shultz PL, Shumsky JS, Galler JR. Development of spatial navigation following prenatal cocaine and malnutrition in rats: Lack of additive effects. Neurotoxicol Teratol 1997;19:363-72.

34. Daniel JM, Roberts SL, Dohanich GP. Effects of ovarian hormones and environment on radial maze and water maze performance of female rats. Physiol Behav 1999;66:11-20. 
35. Healy SD, Braham SR, Braithwaite VA. Proceedings of the royal society of London series B. Biol Sci 1999;266:2303-8.

36. Hunter AJ, Robert, FF. The effect of pirenzepine on spatial learning in the Morris Water Maze. Pharmacol Biochem Behav 1998;30:519-23.

37. Tees RC, Buhrmann k, Hanley J. The effect of early experience on water maze spatial learning and memory in rats. Dev Psychol 1990;23:427-39.

38. Block F, Kunkel M, Schwarz M. Quinolinic acid lesion of the striatum induces impairment in spatial learning and motor performance in rats. Neurosci Lett 1993;149:126-8.

39. Wolfer D, Staglijar-bozecivic M, Lipp HP. Spatial memory and learning in transgenic mice: Fact or artifact? News Physiol Sci 1998; 13:118-23

40. Brandies R, Brandys Y, Yehuda S. The use of the Morris water maze in the study of learning and memory. Int J Neurosci 1989;48:26-9. 